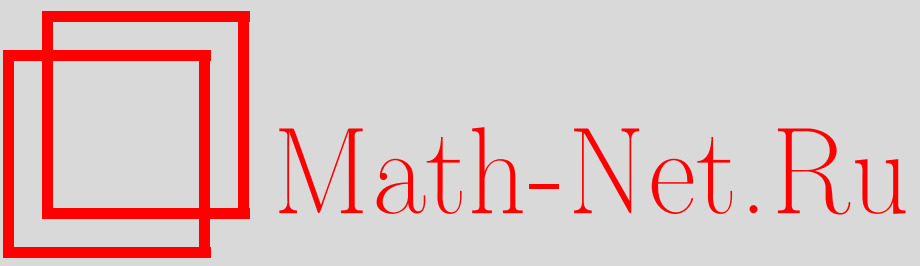

D. V. Belomestny, L. Rüschendorf, M. A. Urusov, Optimal stopping of integral functionals and a "no-loss" free boundary formulation, Теория вероятн. и ее примен., 2009, том 54, выпуск 1, 80-96

DOI: https://doi.org/10.4213/tvp2547

Использование Общероссийского математического портала Math-Net.Ru подразумевает, что вы прочитали и согласны с пользовательским соглашением

http://www. mathnet.ru/rus/agreement

Параметры загрузки:

IP: 54.162 .27 .143

26 апреля 2023 г., 05:01:05 
(C) 2009 г. BELOMESTNY D. V.* RÜSCHENDORF L.**, URUSOV M.A.***

\title{
OPTIMAL STOPPING OF INTEGRAL FUNCTIONALS AND A «NO-LOSS» FREE BOUNDARY FORMULATION ${ }^{1)}$
}

\begin{abstract}
В статье рассматривается модифицированная задача со свободной границей для задачи оптимальной остановки интегральных функционалов от одномерных диффузий. Эта модификация классической задачи со свободной границей была недавно предложена в [21] с целью включить в рассмотрение диффузии с нерегулярными коэффициентами. Основным результатом упомянутой работы является верификационная теорема. Решения модифицированной задачи со свободной границей позволяют найти решения задачи оптимальной остановки. В этой работе мы устанавливаем, что верно и обратное: решения задачи оптимальной остановки являются решениями модифицированной задачи со свободной границей. Таким образом, модифицированная задача со свободной границей не «теряет» решений соответствующей задачи оптимальной остановки. В частности, мы доказываем, что в нашей ситуации гладкое склеивание всегда имеет место. В заключительной части статьи обсуждаются подобные вопросы для подхода, основанного на понятии вязких решений (viscosity solutions), и описывается преимущество модифицированной задачи со свободной границей.
\end{abstract}

Ключевые слова и фразы: оптимальная остановка, задача со свободной границей, одномерная диффузия, условия ЭнгельбертаШмидта, локальное время, формула для времени пребывания, формула Ито-Танака, вязкое решение (viscosity solution) одномерного обыкновенного дифференциального уравнения второго порядка.

1. Introduction. The literature on optimal stopping for diffusions is very rich, also for the one-dimensional case that we consider here. We mention the monographs [23], [12], and [18] for the general theory.

* Weierstrass Institute for Applied Analysis and Stochastics, Mohrenstraße 39, 10117 Berlin, Germany; e-mail: belomest@wias-berlin.de

** Department of Mathematical Stochastics, University of Freiburg, Eckerstraße 1, 79104 Freiburg, Germany; e-mail: ruschen@stochastik.uni-freiburg.de

*** Institute of Mathematics, MA 7-4, Berlin University of Technology, Straße des 17. Juni 136, 10623 Berlin; Quantitative Products Laboratory, Global Markets Equity, Deutsche Bank AG, Alexanderstraße 5, 10178 Berlin, Germany; e-mail: urusov@math.tuberlin.de

1) Supported in part by the SFB 649 «Economic Risk». 
An effective method for solving optimal stopping problems for regular diffusions is to develop a connection with some related free boundary problems of Stefan type. A verification theorem implies that solving the free boundary problem with smooth fit (or related conditions) allows one to establish explicit solutions of the optimal stopping problem in certain cases. Many examples of this type are presented in [18]. In the other direction for some classes of optimal stopping problems for regular diffusion processes with smooth coefficients general existence and regularity results for the corresponding free boundary problems have been established. Some of the results in this direction are quite recent, especially concerning the discussion around the smooth fit principle. The smooth fit principle was first used in [14] to find an explicit solution of a certain problem. Grigelionis and Shiryaev [8] were the first who noticed the necessity of smooth fit for very general Markov processes. Since that time smooth fit became a powerful tool for seeking for explicit solutions of stopping problems used by many authors, and it caused many discussions in the literature because it was not always clear when smooth fit should be used and when it is violated. In the positive direction, let us mention sufficient conditions for smooth fit in [23], [24], and $[22$, p. 96]. In [13, Section 4, Remark 5] there is a related observation that holds in a very general situation. In the negative direction, in $[22$, p. 98 , Example (iii)] and [15, Section 4] (see also [4, Sections 6.7 and 6.11], [18, Chap. IV, Example 9.1]) examples are given, where the smooth fit principle does not hold due to a discontinuity of either the gain function or its derivative at the boundary of the stopping region. From this perspective a striking example is given in [17] or in [18, Chap. IV, Example 9.7], where the gain function is smooth and smooth fit does not hold for a certain regular diffusion. For a more detailed discussion of the whole issue, see [18, Chap. III, Section 7 and Chap. IV, Section 9] and the references therein.

The present paper is concerned with optimal stopping problems for integral functionals of one-dimensional diffusions, possibly with irregular (e.g., discontinuous) coefficients. This irregularity implies that the value function is generally not regular enough to be a solution of the related free boundary problem in the standard form. To deal with this problem we introduce a modified free boundary problem and establish that with this modification results go in both directions: a verification theorem as well as the converse. In particular, smooth fit is proved in our situation of stopping of integral functionals (cf. the discussion above).

More precisely, we consider the following situation. Let $X=\left(X_{t}\right)_{t \in[0, \infty)}$ be a (possibly, explosive) one-dimensional diffusion with state space $J=$ $(\ell, r),-\infty \leqslant \ell<r \leqslant \infty$, that is a weak solution of the SDE

$$
d X_{t}=\mu\left(X_{t}\right) d t+\sigma\left(X_{t}\right) d W_{t},
$$

with $\mathbf{P}_{x}\left(X_{0}=x\right)=1, x \in J$. Here $W$ is a Brownian motion and $\mu$ and 
$\sigma$ are Borel functions $J \rightarrow \mathbf{R}$ specified below. We adopt the convention that $X$ stays in the additional state $\Delta$ after the explosion time $\zeta$, i.e., $\zeta$ is a $[0, \infty]$-valued random variable and

(i) $X$ is $J$-valued and continuous on $[0, \zeta)$;

(ii) if $\zeta<\infty$, then $X \equiv \Delta$ on $[\zeta, \infty)$ and either $\lim _{t \uparrow \zeta} X_{t}=\ell$ or $\lim _{t \uparrow \zeta} X_{t}=r$.

We consider optimal stopping problems of integral functionals of the form

$$
V^{*}(x)=\sup _{\tau \in \mathfrak{M}} \mathbf{E}_{x}\left[\int_{0}^{\tau} e^{-\Lambda_{u}} f\left(X_{u}\right) d u\right], \quad x \in J .
$$

Here $\mathbf{E}_{x}$ denotes the expectation under the measure $\mathbf{P}_{x}$,

$$
\Lambda_{t}=\int_{0}^{t} \lambda\left(X_{u}\right) d u, \quad t \in[0, \infty)
$$

$f$ (respectively, $\lambda$ ) is a Borel function $J \rightarrow \mathbf{R}$ (respectively, $J \rightarrow[0, \infty)$ ) specified below, and $\mathfrak{M}$ is the class of stopping times $\tau$ of $X$ satisfying

$$
\mathbf{E}_{x}\left[\int_{0}^{\tau} e^{-\Lambda_{u}} f^{+}\left(X_{u}\right) d u\right]<\infty \quad \text { or } \quad \mathbf{E}_{x}\left[\int_{0}^{\tau} e^{-\Lambda_{u}} f^{-}\left(X_{u}\right) d u\right]<\infty .
$$

We use the following convention. For any function $g: J \rightarrow \mathbf{R}$, we define $g(\Delta)=0$. In particular, this concerns the functions $f$ and $\lambda$ in (2) and (3).

One encounters concrete stopping problems of type (2) in the literature. For example, in [7] the problem of stopping a Brownian motion as close as possible to its maximum is reduced to a problem of type (2). In order to solve a stochastic control problem the authors of [10] study a stopping problem, that can be reduced to a problem of integral type. It is interesting to note that in [10] the diffusion $X$ is explosive.

In [21] problem (2) is studied via a suitable free boundary approach for functions $f$ having the following form (see Fig. 1): there exist points $x_{1 \ell} \leqslant x_{1 r}<x_{2 \ell} \leqslant x_{2 r}$ in $J$ such that $f=0$ on $\left[x_{1 \ell}, x_{1 r}\right] \cup\left[x_{2 \ell}, x_{2 r}\right], f>0$ on $\left(x_{1 r}, x_{2 \ell}\right)$, and $f<0$ on $\left(\ell, x_{1 \ell}\right) \cup\left(x_{2 r}, r\right)$. In what follows, we say that $f$ has a two-sided form for such functions $f$. For many stopping problems of type (2) in the literature, $f$ has a two-sided form. This is, e.g., the case for the problems of [7] and of [10] mentioned above whenever their parameters belong to some domain (see [21, Remark (ii) in Section 2.1] for a more detailed discussion).

For $a, b \in J, a<b$, we denote by $\tau_{a, b}$ the stopping time

$$
\tau_{a, b}=\inf \left\{t \in[0, \infty): X_{t} \leqslant a \text { or } X_{t} \geqslant b\right\}
$$

(as usual, inf $\varnothing:=\infty$ ). If $f$ has a two-sided form, one may expect that problem (2) has a two-sided optimal stopping time, i.e., an optimal stopping 
time of the form $\tau_{\alpha^{*}, \beta^{*}}$. One can formulate a classical free boundary problem on the triplet $\left(V^{*}, \alpha^{*}, \beta^{*}\right)$ in order to find the value function in (2) and the boundaries $\alpha^{*}$ and $\beta^{*}$ of the stopping region. However, in our situation the functions $\mu, \sigma, f$, and $\lambda$ are allowed to be irregular (e.g., discontinuous), hence, the standard free boundary formulation can lose a two-sided solution of the stopping problem in the following sense: it can happen that there exists a two-sided optimal stopping time but the related free boundary problem has no solutions.

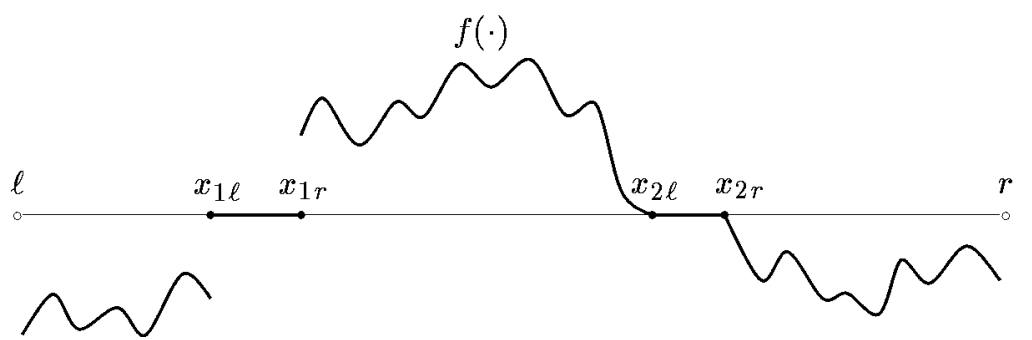

Fig. 1. $f$ has a two-sided form

For this class of stopping problems for diffusions, possibly with irregular coefficients, a modified version of the free boundary has been introduced in the recent paper [21]. The main result in that paper is a verification theorem for this version stating as in the classical regular case the sufficiency of the modified free boundary version. A result in the converse (necessary) direction is announced there. The main contribution of this paper is a general result stating the necessity of the modified free boundary formulation, i.e., the modified free boundary problem does not «lose» solutions of the stopping problem. The proof of this result is technically involved and needs to develop some new tools and techniques which might be of use in further extensions. Both directions together imply that the modified free boundary problem is completely adequate for this type of stopping problems for diffusions, possibly with irregular coefficients. As important practical consequence it is without loss to concentrate on the modified PDE formulation for the solution of this kind of stopping problems. For some explicit examples and a general class of explicitly solvable cases we refer to [21].

We would like also to mention some related papers, [22], [1], [4], [3], [13], where stopping problems of the form $\sup _{\tau} \mathbf{E}_{x}\left[e^{-\Lambda_{\tau}} g\left(X_{\tau}\right) I(\tau<\infty)\right] \gg$ are studied. The authors of this papers allow the coefficients of $X$ as well as the payoff function to be irregular (e.g., discontinuous). In the first four of these papers different approaches are introduced, some general results are obtained, and several examples are treated explicitly. Neither of these approaches is based on the free boundary method - the one we use here. In [13], it is proved that the value function in their problem is the difference of two convex functions and satisfies a certain variational inequality. In our 
situation the value function is even more regular (though the functions $\mu, \sigma$, $f$, and $\lambda$ can be irregular): it is differentiable and its derivative is absolutely continuous (see Theorem 2.1). This agrees with the intuition: the integral functionals $\mathbf{E}_{x}\left[\int_{0}^{\tau} e^{-\Lambda_{u}} f\left(X_{u}\right) d u\right]$ are «more regular» than the functionals $\mathbf{E}_{x}\left[e^{-\Lambda_{\tau}} g\left(X_{\tau}\right) I(\tau<\infty)\right]$. Hence, it is natural to expect that value functions for integral functionals should also be «more regular».

Another difference of the papers mentioned above with our paper is that we consider optimal stopping of integral functionals. Of course, stopping of integral functionals is well established in the literature. For instance, the classical change point problem in Brownian setting reduces to a certain problem of such type (see [23]). For a more general formulation of the problem (with an integral term as well as some other ones) see, e.g., [18, Chap. III, (6.0.1)]. However, the emphasis in our paper is on the point that all the functions involved (the coefficients of the SDE as well as $f$ and $\lambda$ ) can be very irregular. It is this reason that causes us to modify the standard free boundary problem (cf. with the fact that the function $L$ should be continuous in [18, Chap. III, (6.0.1)]).

This paper has the following structure. In Section 2 we introduce our assumptions, formulate the main result (Theorem 2.1), and describe its relation to some results of [21]. Related issues on the viscosity approach are discussed in Section 4. Loosely speaking, the standard free boundary formulation understood in the viscosity sense is not «no-loss» in our setting, which is due to possible discontinuities in the functions $\mu, \sigma, f$, and $\lambda$. If we weaken the notion of viscosity solution a bit (we call this $*$-viscosity solution), then we obtain the «no-loss» property but the verification theorem is not true for $*$-viscosity solutions (compare this with the fact that for our modified free boundary formulation we have both the «no-loss» property and the verification theorem; see Theorems 2.1 and (2.2) below). Finally, Theorem 2.1 is proved in Section 3. In Section 5 we prove a technical lemma used in the proof of that theorem.

2. Main result. At first we describe our assumptions on the functions $\mu, \sigma, f$, and $\lambda$.

A s s u m ption 1. The coefficients $\mu$ and $\sigma$ of the SDE (1) satisfy the Engelbert-Schmidt conditions

$$
\sigma(x) \neq 0 \quad \forall x \in J, \quad \frac{1+|\mu|}{\sigma^{2}} \in L_{\mathrm{loc}}^{1}(J),
$$

where $L_{\text {loc }}^{1}(J)$ denotes the class of functions $J \rightarrow \mathbf{R}$ that are locally integrable on $J$, i.e., integrable on compact subintervals of $J$.

A s s u m p ti o n 2. The functions $f: J \rightarrow \mathbf{R}$ in (2) and $\lambda: J \rightarrow[0, \infty)$ in (3) satisfy the conditions

$$
\frac{f}{\sigma^{2}} \in L_{\mathrm{loc}}^{1}(J), \quad \frac{\lambda}{\sigma^{2}} \in L_{\mathrm{loc}}^{1}(J) .
$$


Note that under Assumption 1 we have $\mathbf{E}_{x} \int_{0}^{\tau_{a, b}}\left|g\left(X_{u}\right)\right| d u<\infty, x, a, b \in$ $J, a<b$, whenever $g: J \rightarrow \mathbf{R}$ is a Borel function such that $g / \sigma^{2} \in L_{\text {loc }}^{1}(J)$. In particular, $\tau_{a, b} \in \mathfrak{M}$ for all $a, b \in J, a<b$. For the proof of this, see Lemma A.3 in [21]. Assumption 1 guarantees that the SDE (1) has a unique in law (possibly, explosive) $J \cup\{\Delta\}$-valued weak solution for any starting point $X_{0}=x \in J$ (see [5], [6] or [11, Chap. 5, Theorem 5.15]). The Engelbert-Schmidt conditions are reasonable weak conditions. For example, if $\mu$ is locally bounded on $J$ and $\sigma$ is locally bounded away from zero on $J$, then they are satisfied. Further we note that due to local integrability of the function $1 / \sigma^{2}$ Assumption 2 holds whenever $f$ and $\lambda$ are locally bounded on $J$. Set $F_{t}=\int_{0}^{t} f\left(X_{u}\right) d u$. Using the occupation times formula (see [19, Chap. VI, Corollary (1.6)]) one can prove that $f / \sigma^{2} \in L_{\text {loc }}^{1}(J)$ (respectively, $\left.\lambda / \sigma^{2} \in L_{\text {loc }}^{1}(J)\right)$ if and only if the process $\left(F_{t}\right)$ (respectively, $\left(\Lambda_{t}\right)$ defined in $(3)$ is well defined and finite a.s. on the stochastic interval $[0, \zeta)$. Therefore, Assumption 2 is reasonable.

In what follows, $\nu_{L}$ denotes the Lebesgue measure on $J$. The main subject in this paper is the following modified free boundary problem, which was introduced in [21].

\section{Modified free boundary problem (MFBP):}

$V^{\prime}$ is absolutely continuous on $[\alpha, \beta]$;

$$
\begin{gathered}
\frac{\sigma^{2}(x)}{2} V^{\prime \prime}(x)+\mu(x) V^{\prime}(x)-\lambda(x) V(x)=-f(x) \quad \text { for } \nu_{L} \text {-a.a. } x \in(\alpha, \beta) ; \\
V(x)=0, \quad x \in J \backslash(\alpha, \beta) ; \\
V_{+}^{\prime}(\alpha)=V_{-}^{\prime}(\beta)=0 .
\end{gathered}
$$

We say that a triplet $(V, \alpha, \beta)$ is a solution of (6)-(9) if $\alpha, \beta \in J, \alpha<\beta$, $V \in C^{1}([\alpha, \beta])$, and the triplet $(V, \alpha, \beta)$ satisfies (6)-(9). In (9), $V_{+}^{\prime}$ and $V_{-}^{\prime}$ denote, respectively, right and left derivatives of $V$. Formally, under $V^{\prime}(\alpha)$ and $V^{\prime}(\beta)$ in (6) one should understand right and left derivatives, respectively. However, (8) and (9) imply that the two-sided derivatives exist at both points.

The main contribution of this paper is the following theorem, which is proved in the next section. It implies that the modified free boundary formulation is also necessary and does not «lose» two-sided solutions. It is of interest to note that this theorem does not need the assumption that the gain function $f$ is of two-sided form.

Theorem 2.1. Suppose that Assumptions 1 and 2 hold. If there exist $\alpha^{*}, \beta^{*} \in J, \alpha^{*}<\beta^{*}$, such that the stopping time $\tau_{\alpha^{*}, \beta^{*}}$ is optimal in (2), then $\left(V^{*}, \alpha^{*}, \beta^{*}\right)$ is a solution of the modified free boundary problem (6)-(9).

$\mathrm{R}$ e $\mathrm{m}$ a r k. In particular, Theorem 2.1 contributes to the extensive discussion in the literature around the smooth fit principle. As a part of Theorem 2.1 we prove that in our situation smooth fit always holds. This 
is different with the stopping problems $\sup _{\tau} \mathbf{E}\left[e^{-\Lambda_{\tau}} g\left(X_{\tau}\right) I(\tau<\infty)\right]$, where a point of discontinuity of $g$ or $g^{\prime}$ may be a boundary point of the stopping region, and smooth fit may be violated at such a point. For examples of such type, see [22], [15], and [18, Chap. IV, Example 9.1].

In the sequel, we denote by $B_{\text {loc }}(x)$ the class of functions that are bounded in a sufficiently small neighborhood of $x$ and by $B_{\text {loc }}(J)$ the class of locally bounded functions on $J$. Theorem 2.1 above is complemented by the following verification theorem, which extends Theorem 2.1 in [21], where the case of constant functions $\lambda$ is considered. If we introduce the condition that $\lambda \in B_{\text {loc }}(J)$, then all arguments of proof in that paper can be extended to this more general case.

Theorem 2.2 (Verification theorem). Suppose that Assumptions 1 and 2 hold and that $f$ has a two-sided form (see Fig. 1). Additionally assume that $\lambda \in B_{\mathrm{loc}}(J)$ and $1 / f \in B_{\mathrm{loc}}(x)$ for all $x \in J \backslash\left(\left[x_{1 \ell}, x_{1 r}\right] \cup\left[x_{2 \ell}, x_{2 r}\right]\right)$. If $(V, \alpha, \beta)$ is a nontrivial solution of the modified free boundary problem (6)-(9) (i.e., $V \not \equiv 0)$, then it is a unique nontrivial solution, $V$ is the value function in (2), i.e., $V=V^{*}$, and $\tau_{\alpha, \beta}$ is a unique optimal stopping time in (2).

$\mathrm{R}$ e $\mathrm{m}$ a r k s. (i) As in [21, Theorem 2.3], also an extended version of the verification Theorem 2.2 for «four-sided» functions with two positivity intervals and corresponding stopping times with two disjoint continuation intervals can be proved for the more general case of discounting then in Theorem 2.2. We remark that Theorem 2.1 of this paper was announced (without proof and details) for the special case of constant discounts $\lambda$ in [21].

(ii) If $x_{1 \ell}<x_{1 r}$ or $x_{2 \ell}<x_{2 r}$, then (6)-(9) has trivial solutions $(0, \alpha, \beta)$ for $\alpha<\beta$ belonging to $\left[x_{1 \ell}, x_{1 r}\right]$ or to $\left[x_{2 \ell}, x_{2 r}\right]$. Therefore, we deal only with nontrivial solutions of (6)-(9) in Theorem 2.2.

(iii) It is interesting to note that we have always strict inequalities $\alpha<$ $x_{1 \ell}$ and $\beta>x_{2 r}$, no matter how large negative values the function $f$ takes to the left from $x_{1 \ell}$ or to the right from $x_{2 r}$ (see [21, Proposition 2.9]).

Finally, we provide two simple examples showing that the assumption that $f$ has a two-sided form is essential for Theorem 2.2.

$\mathrm{E} \mathrm{x}$ a $\mathrm{m} \mathrm{p} \mathrm{l} \mathrm{e} \mathrm{2.1.} \mathrm{Here} \mathrm{we} \mathrm{show} \mathrm{how} \mathrm{to} \mathrm{construct} \mathrm{functions} f$ of a not two-sided form such that the MFBP (6)-(9) loses the uniqueness property, i.e., it will have different nontrivial solutions.

First, consider a function $\bar{f}$ of a two-sided form such that the assumptions of Theorem 2.2 are satisfied and the MFBP (6)-(9) with $\bar{f}$ instead of $f$ has a nontrivial solution $(\bar{V}, \bar{\alpha}, \bar{\beta})$. Now we construct a function $f$ by modifying $\bar{f}$ on $(\bar{\beta}, r)$ in such a way that $f>0$ on some $(\bar{\beta}, \bar{\beta}+\varepsilon)$. Note that Remark (iii) above implies that $f$ does not have a two-sided form. Clearly, $(\bar{V}, \bar{\alpha}, \bar{\beta})$ remains a nontrivial solution of (6)-(9) with this new function $f$. However, denoting by $V^{*}$ the value in (2) for the function $f$, we have $\bar{V} \neq V^{*}$ because $\bar{V}(x)=0<V^{*}(x)$ for $x \in(\bar{\beta}, \bar{\beta}+\varepsilon)$, and, similarly, $\tau_{\bar{\alpha}, \bar{\beta}}$ is not op- 
timal in (2).

If, additionally, $\varepsilon$ is small enough and $f$ takes large negative values on $(\bar{\beta}+\varepsilon, r)$, then a two-sided stopping time $\tau_{\alpha^{*}, \beta^{*}}$ will be optimal in (2). By Theorem 2.1, $\left(V^{*}, \alpha^{*}, \beta^{*}\right)$ is another nontrivial solution of (6)-(9).

$\mathrm{E} \times \mathrm{a} \mathrm{m} \mathrm{ple} \mathrm{2.2.} \mathrm{Let} X$ be a Brownian motion and let $J=\mathbf{R}, \lambda \equiv 0$, and $f(x)=1-2 I(x \in[-1,1])$. Contrary to the previous example, one can easily verify that the MFBP (6)-(9) has a unique solution $(V, \alpha, \beta)$ but $V \neq V^{*} \equiv \infty$.

3. Proof of Theorem 2.1. First, we need several lemmas.

Lemma 3.1. Let $\alpha, \beta \in J, \alpha<\beta$. There exists a function $U:[\alpha, \beta] \rightarrow \mathbf{R}$ such that $U \in C^{1}([\alpha, \beta]), U^{\prime}$ is absolutely continuous on $[\alpha, \beta]$, $\frac{\sigma^{2}(x)}{2} U^{\prime \prime}(x)+\mu(x) U^{\prime}(x)-\lambda(x) U(x)=-f(x) \quad$ for $\nu_{L}$-a.a. $x \in[\alpha, \beta]$,

and $U(\alpha)=U(\beta)=0$.

This lemma is proved in Section 5. In what follows, let $L_{t}^{y}$ denote the local time of $X$ at time $t$ and level $y$.

Lemma 3.2. Let $\mu \equiv 0, \varepsilon>0, x \in J, a_{\varepsilon}:=x-\varepsilon \in J$, and $b_{\varepsilon}:=x+\varepsilon \in J$. Then

$$
\mathbf{E}_{x} L_{\tau_{a_{\varepsilon}, b_{\varepsilon}}}^{y}=\varepsilon-|x-y|, \quad y \in\left(a_{\varepsilon}, b_{\varepsilon}\right),
$$

and

$$
\mathbf{E}_{x}\left(L_{\tau_{a_{\varepsilon}, b_{\varepsilon}}}^{x}\right)^{2} \leqslant c_{0} \varepsilon^{2}
$$

for some constant $c_{0}$ that does not depend on $\varepsilon$ and $x$.

P r o o f. Since $\left(X_{t \wedge \tau_{a_{\varepsilon}, b_{\varepsilon}}}\right)$ is a bounded $\mathbf{P}_{x}$-martingale, it follows that $\mathbf{E}_{x} X_{\tau_{a_{\varepsilon}, b_{\varepsilon}}}=x$. Applying the fact that $\tau_{a_{\varepsilon}, b_{\varepsilon}}<\infty \mathbf{P}_{x^{-}}$-a.s., we get

$$
\mathbf{P}_{x}\left(X_{\tau_{a_{\varepsilon}, b_{\varepsilon}}}=a_{\varepsilon}\right)=\mathbf{P}_{x}\left(X_{\tau_{a_{\varepsilon}, b_{\varepsilon}}}=b_{\varepsilon}\right)=\frac{1}{2} .
$$

For any $y \in\left(a_{\varepsilon}, b_{\varepsilon}\right)$, the Tanaka formula under $\mathbf{P}_{x}$ implies

$$
\left|X_{\tau_{a_{\varepsilon}, b_{\varepsilon}}}-y\right|=|x-y|+\int_{0}^{\tau_{a_{\varepsilon}, b_{\varepsilon}}} \operatorname{sign}\left(X_{u}-y\right) \sigma\left(X_{u}\right) d W_{u}+L_{\tau_{a_{\varepsilon}, b_{\varepsilon}}}^{y} \quad \mathbf{P}_{x^{-} \text {-a.s. }}
$$

where

$$
\text { sign } y= \begin{cases}1 & \text { if } y>0 \\ -1 & \text { if } y \leqslant 0\end{cases}
$$

The process $M_{t}:=\int_{0}^{t \wedge \tau_{a_{\varepsilon}, b_{\varepsilon}}} \operatorname{sign}\left(X_{u}-y\right) \sigma\left(X_{u}\right) d W_{u}$ is a $\mathbf{P}_{x}$-square integrable martingale. Indeed,

$$
[M]_{\infty}=\int_{0}^{\tau_{a_{\varepsilon}, b_{\varepsilon}}} \sigma^{2}\left(X_{u}\right) d u=[X]_{\tau_{a_{\varepsilon}, b_{\varepsilon}}} \quad \mathbf{P}_{x^{-} \text {-a.s. }}
$$


and $\mathbf{E}_{x}[X]_{\tau_{a_{\varepsilon}, b_{\varepsilon}}}<\infty$ because $\left(X_{t \wedge \tau_{a_{\varepsilon}, b_{\varepsilon}}}\right)$ is a bounded $\mathbf{P}_{x}$-martingale. Hence, $\mathbf{E}_{x} M_{\tau_{a_{\varepsilon}, b_{\varepsilon}}}=0$ and (11) follows by computing the expectations in (13). Further, it follows from (13) and the Burkholder-Davis-Gundy inequality that

$$
\begin{aligned}
\mathbf{E}_{x}\left(L_{\tau_{a_{\varepsilon}, b_{\varepsilon}}}^{x}\right)^{2} & =\varepsilon^{2}+\mathbf{E}_{x} M_{\tau_{a_{\varepsilon}, b_{\varepsilon}}}^{2}=\varepsilon^{2}+\mathbf{E}_{x}[X]_{\tau_{a_{\varepsilon}, b_{\varepsilon}}} \\
& \leqslant \varepsilon^{2}+c_{1} \mathbf{E}_{x} \sup _{t \leqslant \tau_{a_{\varepsilon}, b_{\varepsilon}}}\left|X_{t}-x\right|^{2}=\left(1+c_{1}\right) \varepsilon^{2}
\end{aligned}
$$

for some universal constant $c_{1}$. We obtain (12) with $c_{0}=1+c_{1}$. Lemma 3.2 is proved.

In the sequel, we often use the following observation without further explanations: if $A \subseteq J$ with $\nu_{L}(A)=0$, then

$$
\nu_{L}\left(\left\{t \in[0, \infty): X_{t} \in A\right\}\right)=0 \quad \mathbf{P}_{x^{-} \text {-a.s. }, \quad x \in J .}
$$

This can easily be proved using the occupation times formula.

P r o of of $\mathrm{Th}$ e o r e $\mathrm{m}$ 2.1. 1) First, we additionally assume that $\mu \equiv 0$. Let $U:\left[\alpha^{*}, \beta^{*}\right] \rightarrow \mathbf{R}$ be a solution of (10) as in Lemma 3.1 with $\alpha=\alpha^{*}$ and $\beta=\beta^{*}$ and denote

$$
V(x)= \begin{cases}U(x) & \text { if } x \in\left(\alpha^{*}, \beta^{*}\right) \\ 0 & \text { if } x \in J \backslash\left(\alpha^{*}, \beta^{*}\right) .\end{cases}
$$

Our aim is to prove that $V=V^{*}$. If $x \in J \backslash\left(\alpha^{*}, \beta^{*}\right)$, then $V(x)=V^{*}(x)=0$. Suppose that $x \in\left(\alpha^{*}, \beta^{*}\right)$. Since $U^{\prime}$ is absolutely continuous (hence, of bounded variation), we can apply the Itô-Tanaka formula to $U\left(X_{t \wedge \tau_{\alpha^{*}, \beta^{*}}}\right)$ (see [19, Chap. VI, Theorem (1.5)]). We obtain

$$
\begin{aligned}
U\left(X_{t \wedge \tau_{\alpha^{*}, \beta^{*}}}\right)= & U(x)+\int_{0}^{t \wedge \tau_{\alpha^{*}, \beta^{*}}} U^{\prime}\left(X_{u}\right) \sigma\left(X_{u}\right) d W_{u} \\
& +\frac{1}{2} \int_{J} L_{t \wedge \tau_{\alpha^{*}, \beta^{*}}}^{y} U^{\prime}(d y) \quad \mathbf{P}_{x^{-} \text {-a.s. }} \quad t \in[0, \infty) .
\end{aligned}
$$

Since $U^{\prime}$ is absolutely continuous, we have $U^{\prime}(d y)=U^{\prime \prime}(y) d y$. Applying the occupation times formula to the last term on the right-hand side of (14), we find that

$$
\begin{aligned}
& U\left(X_{t \wedge \tau_{\alpha^{*}, \beta^{*}}}\right)=U(x)+\int_{0}^{t \wedge \tau_{\alpha^{*}, \beta^{*}}} U^{\prime}\left(X_{u}\right) \sigma\left(X_{u}\right) d W_{u} \\
& +\int_{0}^{t \wedge \tau_{\alpha^{*}, \beta^{*}}} \frac{\sigma^{2}\left(X_{u}\right)}{2} U^{\prime \prime}\left(X_{u}\right) d u \quad \mathbf{P}_{x^{-} \text {-a.s. }}, \quad t \in[0, \infty) .
\end{aligned}
$$

By the product rule, this implies

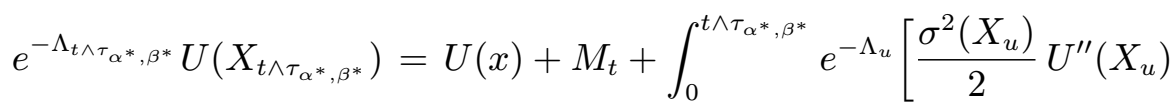

$$
\begin{aligned}
& \left.-\lambda\left(X_{u}\right) U\left(X_{u}\right)\right] d u \quad \mathbf{P}_{x} \text {-a.s., } \quad t \in[0, \infty)
\end{aligned}
$$


where

$$
M_{t}=\int_{0}^{t \wedge \tau_{\alpha^{*}, \beta^{*}}} e^{-\Lambda_{u}} U^{\prime}\left(X_{u}\right) \sigma\left(X_{u}\right) d W_{u} .
$$

The function $U^{\prime}$ is continuous. We have

$$
[M]_{\infty} \leqslant \text { const } \int_{0}^{\tau_{\alpha^{*}, \beta^{*}}} \sigma^{2}\left(X_{u}\right) d u=\text { const }[X]_{\tau_{\alpha^{*}, \beta^{*}}} \quad \mathbf{P}_{x^{-}} \text {a.s. }
$$

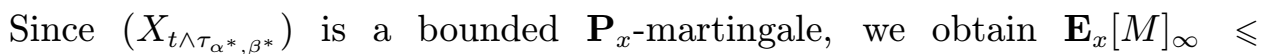
const $\mathbf{E}_{x}[X]_{\tau_{\alpha^{*}, \beta^{*}}}<\infty$. Hence, $\mathbf{E}_{x} M_{\tau_{\alpha^{*}, \beta^{*}}}=0$. Applying relation (10) and $U\left(\alpha^{*}\right)=U\left(\beta^{*}\right)=0$, we obtain from (16) that

$$
U(x)=\mathbf{E}_{x} \int_{0}^{\tau_{\alpha^{*}, \beta^{*}}} e^{-\Lambda_{u}} f\left(X_{u}\right) d u
$$

Then $V(x)=V^{*}(x)$ because $V(x)=U(x)$ and $\tau_{\alpha^{*}, \beta^{*}}$ is an optimal stopping time in (2).

2) We continue to work under the assumption $\mu \equiv 0$. It remains to prove that the boundary condition (9) is fulfilled for $V=V^{*}$. Note that the dynamic programming principle implies that for any $x, a, b \in J, a<b$, we have

$$
V(x) \geqslant \mathbf{E}_{x}\left[e^{-\Lambda_{\tau_{a, b}}} V\left(X_{\tau_{a, b}}\right)+\int_{0}^{\tau_{a, b}} e^{-\Lambda_{u}} f\left(X_{u}\right) d u\right] .
$$

Alternatively, one can prove (17) directly using the strong Markov property. The right derivative $V_{+}^{\prime}\left(\alpha^{*}\right)$ exists and is finite because $V=U$ on $\left[\alpha^{*}, \beta^{*}\right.$. Since $V\left(\alpha^{*}\right)=0 \leqslant V^{*}(x)=V(x), x \in J$, we obtain $V_{+}^{\prime}\left(\alpha^{*}\right) \geqslant 0$. Assuming that $V_{+}^{\prime}\left(\alpha^{*}\right)>0$ we will show that we obtain a contradiction to (17).

For $\varepsilon>0$, we set $a_{\varepsilon}=\alpha^{*}-\varepsilon, b_{\varepsilon}=\alpha^{*}+\varepsilon$ and work below with sufficiently small $\varepsilon$ so that $a_{\varepsilon} \in J$ and $b_{\varepsilon}<\beta^{*}$. Applying the Itô-Tanaka formula under $\mathbf{P}_{\alpha^{*}}$ and proceeding as in (14)-(16), we obtain (note that $V\left(\alpha^{*}\right)=0$ )

$$
\begin{aligned}
& e^{-\Lambda_{\tau_{a_{\varepsilon}, b_{\varepsilon}}} V\left(X_{\tau_{a_{\varepsilon}, b_{\varepsilon}}}\right)}=M_{\tau_{a_{\varepsilon}, b_{\varepsilon}}}+c \int_{0}^{\tau_{a_{\varepsilon}, b_{\varepsilon}}} e^{-\Lambda_{u}} d L_{u}^{\alpha^{*}} \\
& \quad+\int_{0}^{\tau_{a_{\varepsilon}, b_{\varepsilon}}} e^{-\Lambda_{u}}\left[\frac{\sigma^{2}\left(X_{u}\right)}{2} V^{\prime \prime}\left(X_{u}\right)-\lambda\left(X_{u}\right) V\left(X_{u}\right)\right] d u \quad \mathbf{P}_{\alpha^{*} \text {-a.s. }},
\end{aligned}
$$

where $c=V_{+}^{\prime}\left(\alpha^{*}\right) / 2>0$ and $M_{\tau_{a_{\varepsilon}, b_{\varepsilon}}}=\int_{0}^{\tau_{a_{\varepsilon}, b_{\varepsilon}}} e^{-\Lambda_{u}} V_{-}^{\prime}\left(X_{u}\right) \sigma\left(X_{u}\right) d W_{u}$. As earlier, $\mathbf{E}_{\alpha^{*}} M_{\tau_{a_{\varepsilon}, b_{\varepsilon}}}=0$. The term $c \int_{0}^{\tau_{\tau_{\varepsilon}, b_{\varepsilon}}} e^{-\Lambda_{u}} d L_{u}^{\alpha^{*}}$ in (18) appears due to the fact that the function $V^{\prime}$ has discontinuities at $\alpha^{*}$ and $\beta^{*}$. Hence, the measure $V^{\prime}(d y)$ (with the distribution function $V_{+}^{\prime}$ ) appearing in the ItôTanaka formula has the form $V^{\prime}(d y)=V^{\prime \prime}(y) d y+V_{+}^{\prime}\left(\alpha^{*}\right) \delta_{\alpha^{*}}-V_{-}^{\prime}\left(\beta^{*}\right) \delta_{\beta^{*}}$, where $\delta_{x}$ denotes the unit measure concentrated at the point $x$. Note that the local time at level $\beta^{*}$ does not appear in (18) because $L^{\beta^{*}}=0 \mathbf{P}_{\alpha^{*}}$-a.s. on $\left[0, \tau_{a_{\varepsilon}, b_{\varepsilon}}\right]$ due to $b_{\varepsilon}<\beta^{*}$. 
We have $\left(\sigma^{2} / 2\right) V^{\prime \prime}-\lambda V=-f I_{\left(\alpha^{*}, \beta^{*}\right)} \nu_{L^{-}}$a.e. on $J$. Hence, it follows from (18) that

$$
\begin{aligned}
\mathbf{E}_{\alpha^{*}} & {\left[e^{-\Lambda_{\tau_{a_{\varepsilon}}, b_{\varepsilon}}} V\left(X_{\tau_{a_{\varepsilon}, b_{\varepsilon}}}\right)+\int_{0}^{\tau_{a_{\varepsilon}, b_{\varepsilon}}} e^{-\Lambda_{u}} f\left(X_{u}\right) d u\right] } \\
& =\mathbf{E}_{\alpha^{*}}\left[c \int_{0}^{\tau_{a_{\varepsilon}, b_{\varepsilon}}} e^{-\Lambda_{u}} d L_{u}^{\alpha^{*}}+\int_{0}^{\tau_{a_{\varepsilon}, b_{\varepsilon}}} e^{-\Lambda_{u}} h\left(X_{u}\right) d u\right],
\end{aligned}
$$

where $h:=f I_{J \backslash\left(\alpha^{*}, \beta^{*}\right)}$. Putting $g:=|h|$, we get

$$
\begin{aligned}
\int_{0}^{\tau_{a_{\varepsilon}, b_{\varepsilon}}} e^{-\Lambda_{u}} h\left(X_{u}\right) d u & \geqslant-\int_{0}^{\tau_{a_{\varepsilon}, b_{\varepsilon}}} g\left(X_{u}\right) d u \\
& =-\int_{a_{\varepsilon}}^{b_{\varepsilon}} \frac{g(y)}{\sigma^{2}(y)} L_{\tau_{a_{\varepsilon}, b_{\varepsilon}}}^{y} d y \quad \mathbf{P}_{\alpha^{*} \text {-a.s. }},
\end{aligned}
$$

where the last equality follows from the occupation times formula. It follows from (19), $g \geqslant 0$, and Lemma 3.2 that

$$
\begin{aligned}
& \mathbf{E}_{\alpha^{*}}\left[e^{-\Lambda_{\tau_{a_{\varepsilon}}, b_{\varepsilon}}} V\left(X_{\tau_{a_{\varepsilon}, b_{\varepsilon}}}\right)+\int_{0}^{\tau_{a_{\varepsilon}, b_{\varepsilon}}} e^{-\Lambda_{u}} f\left(X_{u}\right) d u\right] \\
& \geqslant c \mathbf{E}_{\alpha^{*}}\left[e^{-\Lambda_{\tau_{a_{\varepsilon}, b_{\varepsilon}}}} L_{\tau_{a_{\varepsilon}, b_{\varepsilon}}}^{\alpha^{*}}\right]-\int_{a_{\varepsilon}}^{b_{\varepsilon}} \frac{g(y)}{\sigma^{2}(y)} \mathbf{E}_{\alpha^{*}} L_{\tau_{a_{\varepsilon}, b_{\varepsilon}}}^{y} d y \\
& \geqslant c \varepsilon-c \mathbf{E}_{\alpha^{*}}\left[\left(1-e^{-\Lambda_{\tau_{a_{\varepsilon}, b_{\varepsilon}}}}\right) L_{\tau_{a_{\varepsilon}, b_{\varepsilon}}}^{\alpha^{*}}\right]-\varepsilon \int_{a_{\varepsilon}}^{b_{\varepsilon}} \frac{g(y)}{\sigma^{2}(y)} d y \\
& =\left(c-\int_{a_{\varepsilon}}^{b_{\varepsilon}} \frac{g(y)}{\sigma^{2}(y)} d y\right) \varepsilon-c \mathbf{E}_{\alpha^{*}}\left[\left(1-e^{-\Lambda_{\tau_{a_{\varepsilon}, b_{\varepsilon}}}}\right) L_{\tau_{a_{\varepsilon}, b_{\varepsilon}}}^{\alpha^{*}}\right] .
\end{aligned}
$$

Now we fix an arbitrary $\delta \in(0,1)$ and define $T_{\delta}=\inf \left\{t \in[0, \infty): \Lambda_{t}>\right.$ $-\ln (1-\delta)\}(\inf \varnothing:=\infty)$. By the occupation times formula,

$$
\Lambda_{t}=\int_{0}^{t} \lambda\left(X_{u}\right) d u=\int_{J} \frac{\lambda(y)}{\sigma^{2}(y)} L_{t}^{y} d y<\infty \quad \mathbf{P}_{\alpha^{*}-\text { a.s. on }\{t<\zeta\}}
$$

because $\lambda / \sigma^{2} \in L_{\text {loc }}^{1}(J)$ and $\mathbf{P}_{\alpha^{*}}$-a.s. on $\{t<\zeta\}$ the function $y \mapsto L_{t}^{y}$ has a compact support in $J$ and is bounded due to the fact that it is càdlàg (recall

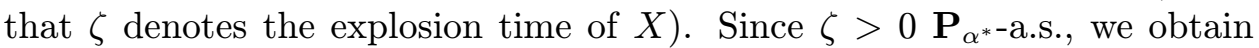
that for $\mathbf{P}_{\alpha^{*}}$-a.a. elementary outcomes $\omega$ there exists $t_{0}=t_{0}(\omega)$ such that $\Lambda_{t_{0}}<\infty$. Due to the continuity of $\left(\Lambda_{u}\right)_{u \in\left[0, t_{0}\right]}$ we get $\Lambda_{t} \downarrow 0 \mathbf{P}_{\alpha^{*}}$-a.s. as $t \downarrow 0$, hence, $T_{\delta}>0 \mathbf{P}_{\alpha^{*}}$-a.s.

We have

$$
\left(1-e^{-\Lambda_{\tau_{a_{\varepsilon}, b_{\varepsilon}}}}\right) L_{\tau_{a_{\varepsilon}, b_{\varepsilon}}}^{\alpha^{*}} \leqslant \delta L_{\tau_{a_{\varepsilon}, b_{\varepsilon}}^{*}}^{\alpha^{*}}+L_{\tau_{a_{\varepsilon}, b_{\varepsilon}}}^{\alpha^{*}} I\left(\tau_{a_{\varepsilon}, b_{\varepsilon}}>T_{\delta}\right) .
$$

Hence, by Lemma 3.2,

$$
\mathbf{E}_{\alpha^{*}}\left[\left(1-e^{-\Lambda_{\tau_{a_{\varepsilon}, b_{\varepsilon}}}}\right) L_{\tau_{a_{\varepsilon}, b_{\varepsilon}}}^{\alpha^{*}}\right] \leqslant \delta \varepsilon+\mathbf{E}_{\alpha^{*}}\left[L_{\tau_{a_{\varepsilon}, b_{\varepsilon}}}^{\alpha^{*}} I\left(\tau_{a_{\varepsilon}, b_{\varepsilon}}>T_{\delta}\right)\right] .
$$


By the Cauchy-Bunyakovski-Schwarz inequality and (12),

$$
\left\{\mathbf{E}_{\alpha^{*}}\left[L_{\tau_{a_{\varepsilon}, b_{\varepsilon}}}^{\alpha^{*}} I\left(\tau_{a_{\varepsilon}, b_{\varepsilon}}>T_{\delta}\right)\right]\right\}^{2} \leqslant c_{0} \varepsilon^{2} \mathbf{P}_{\alpha^{*}}\left(\tau_{a_{\varepsilon}, b_{\varepsilon}}>T_{\delta}\right)
$$

Since $\tau_{a_{\varepsilon}, b_{\varepsilon}} \downarrow 0 \mathbf{P}_{\alpha^{*}}$-a.s. as $\varepsilon \downarrow 0$, and $T_{\delta}>0 \mathbf{P}_{\alpha^{*}}$-a.s., it follows from (21), (22) that

$$
\mathbf{E}_{\alpha^{*}}\left[\left(1-e^{-\Lambda_{\tau_{a_{\varepsilon}, b_{\varepsilon}}}}\right) L_{\tau_{a_{\varepsilon}, b_{\varepsilon}}}^{\alpha^{*}}\right] \leqslant 2 \delta \varepsilon
$$

for sufficiently small $\varepsilon>0$. Since $\delta \in(0,1)$ is arbitrary and $g / \sigma^{2} \in L_{\text {loc }}^{1}(J)$, the right-hand side of (20) is strictly positive for sufficiently small $\varepsilon>0$. This contradicts (17) with $x=\alpha^{*}$. Hence, $V_{+}^{\prime}\left(\alpha^{*}\right)=0$. Similarly, $V_{-}^{\prime}\left(\beta^{*}\right)=0$.

$3)$ Finally we prove the result without the assumption $\mu \equiv 0$. For some fixed $c \in J$ we consider the scale function of the process $X$

$$
p(x)=\int_{c}^{x} \exp \left(-\int_{c}^{y} \frac{2 \mu(z)}{\sigma^{2}(z)} d z\right) d y, \quad x \in J
$$

We define the process $\widetilde{X}_{t}=p\left(X_{t}\right), p(\Delta):=\Delta$, with the state space $\widetilde{J} \cup\{\Delta\}$, $\widetilde{J}=(\widetilde{\ell}, \widetilde{r}):=(p(\ell), p(r))$. Then we have $d \widetilde{X}_{t}=\widetilde{\sigma}\left(\widetilde{X}_{t}\right) d W_{t}$ with $\widetilde{\sigma}(x)=$ $\left(p^{\prime} \sigma\right) \circ p^{-1}(x), x \in \widetilde{J}$. We shall use the alternative notation $\widetilde{\mathbf{P}}_{x}$ for the measure $P_{p^{-1}(x)}$ so that $\widetilde{\mathbf{P}}_{x}\left(\widetilde{X}_{0}=x\right)=1$. Consider now the stopping problem

$$
\widetilde{V}^{*}(x)=\sup _{\tau \in \mathfrak{M}} \widetilde{\mathbf{E}}_{x}\left[\int_{0}^{\tau} e^{-\widetilde{\Lambda}_{u}} \widetilde{f}\left(\widetilde{X}_{u}\right) d u\right], \quad x \in \widetilde{J},
$$

where $\tilde{f}=f \circ p^{-1}, \widetilde{\lambda}=\lambda \circ p^{-1}$, and $\Lambda_{t}=\int_{0}^{t} \widetilde{\lambda}\left(\widetilde{X}_{u}\right) d u$. This stopping problem is a reformulation of problem (2) in the sense that $\widetilde{V}^{*}=V^{*} \circ p^{-1}$, and a stopping time $\tau^{*}$ is optimal in problem $V^{*}(x)$ if and only if it is optimal in problem $\widetilde{V}^{*}(p(x))$. Note that Assumptions 1 and 2 for the functions $\widetilde{\mu} \equiv 0, \widetilde{\sigma}$, $\widetilde{f}$, and $\widetilde{\lambda}$ are satisfied (one should replace $J$ with $\widetilde{J}$ in these conditions). One can easily verify that the triplet $(V, \alpha, \beta)$ is a solution of $(6)-(9)$ if and only if the triplet $(\widetilde{V}, \widetilde{\alpha}, \widetilde{\beta}):=\left(V \circ p^{-1}, p(\alpha), p(\beta)\right)$ is a solution of the modified free boundary problem

$$
\begin{aligned}
& \widetilde{V}^{\prime} \text { is absolutely continuous on }[\widetilde{\alpha}, \widetilde{\beta}] ; \\
& \frac{\widetilde{\sigma}^{2}(x)}{2} \widetilde{V}^{\prime \prime}(x)-\widetilde{\lambda}(x) \widetilde{V}(x)=-\widetilde{f}(x) \quad \text { for } \nu_{L^{-}} \text {a.a. } x \in(\widetilde{\alpha}, \widetilde{\beta}) ; \\
& \widetilde{V}(x)=0, \quad x \in \widetilde{J} \backslash(\widetilde{\alpha}, \widetilde{\beta}) ; \quad \widetilde{V}_{+}^{\prime}(\widetilde{\alpha})=\widetilde{V}_{-}^{\prime}(\widetilde{\beta})=0 .
\end{aligned}
$$

Now the result follows from parts 1) and 2). Theorem 2.1 is proved.

4. Viscosity approach. The reason why we consider the modification (6)-(9) of the classical free boundary problem is that we want to allow irregular $\mu, \sigma, f$, and $\lambda$. Hence, the value function in (2) is not regular enough to be a solution of the free boundary problem in the classical form. A usual 
approach to handle this problem is to consider viscosity solutions (see [2]). It is usually proved that value functions of certain classes of (multidimensional) stopping problems satisfy corresponding variational inequalities in the viscosity sense (see, e.g., [15] or [16]). However, the diffusion coefficients and the payoff are usually assumed to be continuous. In this paper, we consider a modified free boundary formulation rather than variational inequalities. So it is interesting to see, what we obtain for our stopping problem (2), when we consider viscosity solutions for the classical free boundary problem.

Thus, we consider the following free boundary problem:

$$
\begin{aligned}
& \frac{\sigma^{2}(x)}{2} V^{\prime \prime}(x)+\mu(x) V^{\prime}(x)-\lambda(x) V(x)=-f(x), \quad x \in(\alpha, \beta) ; \\
& V(x)=0, \quad x \in J \backslash(\alpha, \beta) ; \\
& V_{+}^{\prime}(\alpha)=V_{-}^{\prime}(\beta)=0
\end{aligned}
$$

and define its viscosity solution as follows (cf. [15, Definition 2.1] for the case of variational inequalities).

D e f i n it i o n 4.1. A viscosity solution of (23)-(25) is a triplet $(V, \alpha, \beta)$ such that $\alpha, \beta \in J, \alpha<\beta, V$ is a continuous function $J \rightarrow \mathbf{R}$ satisfying (24) and (25), and $V$ is both a viscosity subsolution and a viscosity supersolution of $(23)$ in the sense of the following definition.

$\mathrm{D}$ e f i n it i o n 4.2 . Let $\alpha, \beta \in J, \alpha<\beta$. Set $I=(\alpha, \beta)$. A continuous function $V: I \rightarrow \mathbf{R}$ is a viscosity subsolution of (23) if for each $\psi \in C^{2}(I)$ and each $y_{0} \in I$ such that $\psi \geqslant V$ on $I$ and $\psi\left(y_{0}\right)=V\left(y_{0}\right)$ we have

$$
-\frac{\sigma^{2}\left(y_{0}\right)}{2} \psi^{\prime \prime}\left(y_{0}\right)-\mu\left(y_{0}\right) \psi^{\prime}\left(y_{0}\right)+\lambda\left(y_{0}\right) \psi\left(y_{0}\right)-f\left(y_{0}\right) \leqslant 0 .
$$

A continuous function $V: I \rightarrow \mathbf{R}$ is a viscosity supersolution of (23) if for each $\phi \in C^{2}(I)$ and each $y_{0} \in I$ such that $\phi \leqslant V$ on $I$ and $\phi\left(y_{0}\right)=V\left(y_{0}\right)$ we have

$$
-\frac{\sigma^{2}\left(y_{0}\right)}{2} \phi^{\prime \prime}\left(y_{0}\right)-\mu\left(y_{0}\right) \phi^{\prime}\left(y_{0}\right)+\lambda\left(y_{0}\right) \phi\left(y_{0}\right)-f\left(y_{0}\right) \geqslant 0 .
$$

Now we consider the following question.

$\mathrm{Qu}$ e s t i o n 1. Is it true that under the assumptions of Theorem 2.1 the triplet $\left(V^{*}, \alpha^{*}, \beta^{*}\right)$ is a viscosity solution of $(23)-(25)$ ?

The answer is No. The reason for that are possible discontinuities in $\mu, \sigma, f$, and $\lambda$. Indeed, if we change the functions $\mu, \sigma, f$, and $\lambda$ on sets of $\nu_{L}$-measure 0 , then $X$ remains a solution of (1) and problem (2) does not change. A viscosity solution of (23)-(25), however, can lose this property under such a transformation (see (26) and (27)). Suppose that the answer to Question 1 is Yes. Then considering any $\mu, \sigma, f$, and $\lambda$ such that there exists a two-sided optimal stopping time $\tau_{\alpha^{*}, \beta^{*}}$ in (2), we obtain that $\left(V^{*}, \alpha^{*}, \beta^{*}\right)$ is a viscosity solution of $(23)-(25)$. Then we take any appropriate pair $\left(\psi, y_{0}\right)$ of 
Definition 4.2 and modify $f$ only at point $y_{0}$ in order to violate (26). Hence, $\left(V^{*}, \alpha^{*}, \beta^{*}\right)$ is no more a viscosity solution of $(23)-(25)$ with the modified function $f$. This contradicts our assumption that the answer to Question 1 is Yes.

Thus, in order to obtain a positive result in this direction it is natural to define $*$-viscosity solutions of (23)-(25) through $*$-viscosity subsolutions and *-viscosity supersolutions as in Definition 4.1. Here *-viscosity subsolutions and $*$-viscosity supersolutions are defined as follows.

$\mathrm{D}$ e f in ition 4.3. Let $\alpha, \beta \in J, \alpha<\beta$. Set $I=(\alpha, \beta)$. A continuous function $V: I \rightarrow \mathbf{R}$ is a $*$-viscosity subsolution of (23) if for $\nu_{L^{-}}$-a.a. $y_{0} \in I$ the following condition holds: For each $\psi \in C^{2}(I)$ such that $\psi \geqslant V$ on $I$ and $\psi\left(y_{0}\right)=V\left(y_{0}\right)$, condition (26) is satisfied.

A $*$-viscosity supersolution of (23) is defined in a symmetric way.

Now the positive answer to the modification of Question 1 with *viscosity solutions instead of viscosity solutions follows directly from Theorem 2.1.

Corollary 4.1. Suppose that Assumptions 1 and 2 hold. If there exist $\alpha^{*}, \beta^{*} \in J, \alpha^{*}<\beta^{*}$, such that the stopping time $\tau_{\alpha^{*}, \beta^{*}}$ is optimal in (2), then $\left(V^{*}, \alpha^{*}, \beta^{*}\right)$ is a $*$-viscosity solution of $(23)-(25)$.

Then it is natural to pose the following question.

$\mathrm{Qu}$ e stion 2. Suppose that the assumptions of Theorem 2.2 are satisfied and $(V, \alpha, \beta)$ is a nontrivial $*$-viscosity solution of $(23)-(25)$.

(a) Does this imply that $V=V^{*}$ ?

(b) Does this imply that $\tau_{\alpha, \beta}$ is an optimal stopping time in (2)?

In the case of positive answers one could use the standard free boundary $(23)-(25)$ (understood in the $*$-viscosity sense) to solve (2) even with irregular (e.g., discontinuous) functions $\mu, \sigma, f$, and $\lambda$. Unfortunately, the answer is No both to (a) and to (b) as the following examples show.

$\mathrm{E} \times \mathrm{a} \mathrm{m} \mathrm{p} \mathrm{le} \mathrm{4.1.} \mathrm{We} \mathrm{set} \mu \equiv 0, \lambda \equiv 0$ and consider $\sigma$ and $f$ satisfying the assumptions of Theorem 2.2 such that there exists a nontrivial solution $(V, \alpha, \beta)$ of (6)-(9) (see [21, Section 3] for necessary and sufficient conditions). Then by Theorem 2.2, $V$ is the value function in (2), i.e., $V=V^{*}$. We take any continuous function $h: J \rightarrow \mathbf{R}$ such that

$$
\begin{aligned}
h & =0 \text { on } J \backslash(\alpha, \beta), \quad h_{+}^{\prime}(\alpha)=h_{-}^{\prime}(\beta)=0, \\
h^{\prime} & =0 \nu_{L} \text {-a.e. on }(\alpha, \beta), \quad \int_{\alpha}^{\beta} h(x) d x=0,
\end{aligned}
$$

and $h$ is not absolutely continuous on $[\alpha, \beta]$ (such a function $h$ can easily be constructed through the Cantor staircase function) and set

$$
\tilde{V}(y)=V(y)+\int_{\ell}^{y} h(x) d x, \quad y \in J .
$$


Then the triplet $(\widetilde{V}, \alpha, \beta)$ satisfies $(7)-(9)$, hence, $(\widetilde{V}, \alpha, \beta)$ is a nontrivial $*$-viscosity solution of (23)-(25) but $\widetilde{V} \neq V=V^{*}$. As consequence, we obtain a negative answer to part (a) of Question 2.

$\mathrm{E} \times \mathrm{a} \mathrm{m} \mathrm{p} \mathrm{l} \mathrm{e} \mathrm{4.2.} \mathrm{As} \mathrm{earlier,} \mathrm{we} \mathrm{set} \mu \equiv 0, \lambda \equiv 0$ and consider $\sigma$ and $f$ satisfying the assumptions of Theorem 2.2 such that $x_{1 \ell}<x_{1 r}$ and that there exists a nontrivial solution $(V, \alpha, \beta)$ of $(6)-(9)$. Then by Theorem 2.2 , $\tau_{\alpha, \beta}$ is a unique optimal stopping time in (2). However, for any $\widetilde{\alpha}<\widetilde{\beta}$ in $\left[x_{1 \ell}, x_{1 r}\right]$, we can construct a nontrivial $*$-viscosity solution $(\widetilde{V}, \widetilde{\alpha}, \widetilde{\beta})$ of $(23)$ (25) using exactly the same idea as in the previous example. We have $\alpha<$ $\widetilde{\alpha}<\widetilde{\beta}<\beta$ (see Remark (iii) after Theorem 2.2). Hence, the stopping time $\tau_{\tilde{\alpha}, \tilde{\beta}}$ is not optimal in (2). Thus, we obtain a negative answer also to part (b) of Question 2.

5. Appendix. Here we outline a probabilistic proof of Lemma 3.1. Without loss of generality we assume that $\mu \equiv 0$. First, we consider the homogeneous ODE

$$
\frac{\sigma^{2}(x)}{2} U^{\prime \prime}(x)-\lambda(x) U(x)=0 .
$$

Our aim is to prove that there exist two differentiable functions $\phi$ and $\psi$ on $[\alpha, \beta]$ with absolutely continuous derivatives that solve $(28) \nu_{L}$-a.e. on $[\alpha, \beta]$ and satisfy the inequality

$$
\psi(\alpha) \phi(\beta)-\psi(\beta) \phi(\alpha)>0 .
$$

Under the additional assumption that $X$ is nonexplosive (and some technical assumptions on the function $\lambda$ ), this result can be found in [9] or derived from [20, Chap. V, Proposition (50.3)]. Below we follow the lines of [20] and concentrate mostly on the part of the proof that should be done differently (this difference occurs because $X$ can explode). If $\lambda=0 \nu_{L}$-a.e. on $[\alpha, \beta]$, then we can put $\psi(x)=1$ and $\phi(x)=x$. Therefore, below we suppose that $\nu_{L}(\{x \in[\alpha, \beta]: \lambda(x)>0\})>0$. We take two additional points $\alpha^{\prime} \in(\ell, \alpha)$ and $\beta^{\prime} \in(\beta, r)$ and define

$$
\begin{array}{ll}
\psi(x)=\mathbf{E}_{x}\left[e^{-\Lambda_{\tau_{\alpha, \beta^{\prime}}}}\right], & x \in\left[\alpha, \beta^{\prime}\right], \\
\phi(x)=\mathbf{E}_{x}\left[e^{-\Lambda_{\tau_{\alpha^{\prime}, \beta}}}\right], & x \in\left[\alpha^{\prime}, \beta\right] .
\end{array}
$$

One can see that $\psi$ and $\phi$ are continuous and satisfy (29). Consider now the function $\psi$ in more detail. For any $x \in\left[\alpha, \beta^{\prime}\right]$ the Markov property implies

$$
\begin{aligned}
& \mathbf{E}_{x}\left[e^{-\Lambda_{\tau_{\alpha, \beta^{\prime}}}} \mid \mathscr{F}_{t}\right]=e^{-\Lambda_{\tau, \beta^{\prime}}} I\left(\tau_{\alpha, \beta^{\prime}} \leqslant t\right)+\mathbf{E}_{x}\left[e^{-\Lambda_{\tau_{\alpha, \beta^{\prime}}} I\left(\tau_{\alpha, \beta^{\prime}}>t\right) \mid \mathscr{F}_{t}}\right]
\end{aligned}
$$

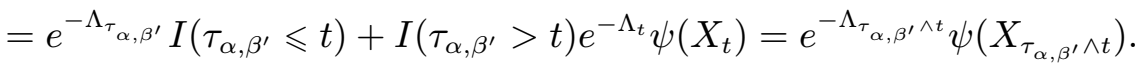


Hence, the process

$$
M_{t}=e^{-\Lambda_{\tau_{\alpha, \beta^{\prime}} \wedge t}} \psi\left(X_{\tau_{\alpha, \beta^{\prime}} \wedge t}\right)
$$

is a uniformly integrable $\mathbf{P}_{x}$-martingale, $x \in\left[\alpha, \beta^{\prime}\right]$. Consequently, for any $x, a, b \in\left[\alpha, \beta^{\prime}\right]$ such that $a<x<b$ it holds that $\mathbf{E}_{x} M_{\tau_{a, b}}=\psi(x)$. Now we are able to prove convexity of $\psi$. Indeed,

$$
\psi(x)=\mathbf{E}_{x}\left[e^{-\Lambda_{\tau_{a, b}}} \psi\left(X_{\tau_{a, b}}\right)\right] \leqslant \mathbf{E}_{x}\left[\psi\left(X_{\tau_{a, b}}\right)\right]=\psi(a) \frac{b-x}{b-a}+\psi(b) \frac{x-a}{b-a} .
$$

Applying the Itô-Tanaka formula to (30), one derives that $\psi$ is differentiable on $\left[\alpha, \beta^{\prime}\right]$ and $\psi^{\prime}$ is absolutely continuous on $\left[\alpha, \beta^{\prime}\right]$. Moreover,

$$
\frac{\sigma^{2}}{2} \psi^{\prime \prime}(x)-\lambda(x) \psi(x)=0
$$

for $\nu_{L}$-a.a. $x \in\left[\alpha, \beta^{\prime}\right]$. The analogous result (with $\left[\alpha, \beta^{\prime}\right]$ replaced by $\left[\alpha^{\prime}, \beta\right]$ ) can be proved for $\phi$ as well.

We turn to the inhomogeneous equation

$$
\frac{\sigma^{2}(x)}{2} U^{\prime \prime}(x)-\lambda(x) U(x)=-f(x)
$$

considered $\nu_{L}$-a.e. on $[\alpha, \beta]$. One can see that the function $\psi^{\prime} \phi-\psi \phi^{\prime}$ is constant on $[\alpha, \beta]$ (cf. $[9$, formula (26)]). We denote this constant by $A$ and note that $A \neq 0$. (Indeed, we can choose $\beta^{\prime}$ close enough to $\beta$ so that $\psi$ attains its minimum at a point $c \in[\alpha, \beta]$. Then $\psi^{\prime}(c) \phi(c)-\psi(c) \phi^{\prime}(c)=$ $-\psi(c) \phi^{\prime}(c) \neq 0$.) A special solution of $(31)$ can be found in the form

$$
U_{0}(x)=\frac{2 \phi(x)}{A} \int_{\alpha}^{x} \frac{\psi(y) f(y) d y}{\sigma^{2}(y)}+\frac{2 \psi(x)}{A} \int_{x}^{\beta} \frac{\phi(y) f(y) d y}{\sigma^{2}(y)}, \quad x \in[\alpha, \beta]
$$

(cf. [9, formula (31)]. Note that since $\alpha>\ell$ and $\beta<r$, both integrals are finite). One can see that the function $U_{0}$ is differentiable everywhere on $[\alpha, \beta]$ and its derivative is absolutely continuous. Finally, due to (29) we can find constants $C_{1}$ and $C_{2}$ such that the function

$$
U(x)=U_{0}(x)+C_{1} \psi(x)+C_{2} \phi(x), \quad x \in[\alpha, \beta],
$$

satisfies $U(\alpha)=U(\beta)=0$. This function $U$ is what we need.

Acknowledgments. We are grateful to several participants of the Second AMaMeF Conference that took place in Bedlewo, Poland, on April 30May 5, 2007, for interesting questions and comments. Especially, we express deep thanks to M. Zervos for an essential idea for the proof of Theorem 2.1. We thank A. N. Shiryaev for several comments that improved the final version of the paper. 


\section{REFERENCES}

1. Beibel M., Lerche H.R. A note on optimal stopping of regular diffusions under random discounting. - Теория вероятн. и ее примен., 2000, т. 45, в. 4, с. 657-669.

2. Crandall M. G., Ishii H., Lions P.-L. User's guide to viscosity solutions of second order partial differential equations. - Bull. Amer. Math. Soc., 1992, v. 27, № 1, p. 1-67.

3. Dayanik $S$. Optimal stopping of linear diffusions with random discounting. - Math. Oper. Res., 2008, v. 33, № 3, p. 645-661.

4. Dayanik S., Karatzas I. On the optimal stopping problem for one-dimensional diffusions. - Stochastic Process. Appl., 2003, v. 107, № 2, p. 173-212.

5. Engelbert H. J., Schmidt W. On one-dimensional stochastic differential equations with generalized drift. - Stochastic Differential Systems (Marseille-Luminy, 1984). Berlin: Springer, 1985, p. 143-155. (Lecture Notes in Control and Inform. Sci., v. 69.)

6. Engelbert H. J., Schmidt W. Strong Markov continuous local martingales and solutions of one-dimensional stochastic differential equations. III. - Math. Nachr., 1991, v. 151, p. 149-197.

7. Graversen S.E., Peskir G., Shiryaev A.N. Stopping Brownian motion without anticipation as close as possible to its ultimate maximum. - Теория вероятн. и ее примен., 2000, т. 45 , в. 1, с. $125-136$.

8. Григелионис Б.И., Ширяев А.Н. О задаче Стефана и оптимальных правилах остановки марковских процессов. - Теория вероятн. и ее примен., 1966, т. 11, B. 4, c. $612-631$.

9. Johnson T.C., Zervos M. The solution to a second order linear ordinary differential equation with a non-homogeneous term that is a measure. - Stochastics, 2007, v. 79, № 3-4, p. 363-382.

10. Karatzas I., Ocone D. A leavable bounded-velocity stochastic control problem. Stochastic Process. Appl., 2002, v. 99, № 1, p. 31-51.

11. Karatzas I., Shreve S.E. Brownian Motion and Stochastic Calculus. New York: Springer-Verlag, 1991, 470 p. (Graduate Texts in Math., v. 113.)

12. Крылов Н. В. Управляемые процессы диффузионного типа. М.: Наука, 1977, $399 \mathrm{c}$.

13. Lamberton D., Zervos $M$. On the problem of optimally stopping a one-dimensional Itô diffusion. Preprint. London: The London School of Economics and Political Science, 2006; http://www.maths.lse.ac.uk/Personal/mihail/publications/

14. Михалевич B. С. Байесовский выбор между двумя гипотезами о среднем значении нормального процесса. - Вестн. Киев. ун-та, 1958, № 1, с. 101-104.

15. Øksendal B., Reikvam K. Viscosity solutions of optimal stopping problems. Stochastics Stochastics Rep., 1998, v. 62, № 3-4, p. 285-301.

16. Øksendal B., Sulem A. Applied Stochastic Control of Jump Diffusions. Berlin: Springer-Verlag, 2005, 208 p.

17. Peskir G. Principle of smooth fit and diffusions with angles. - Stochastics, 2007, v. 79 , № 3-4, p. 293-302.

18. Peskir G., Shiryaev A.N. Optimal Stopping and Free-Boundary Problems. Basel: Birkhäuser, 2006, 500 p.

19. Revuz D., Yor M. Continuous Martingales and Brownian Motion. Berlin: SpringerVerlag, 1999, 602 p. (Grundlehren Math. Wiss., v. 293.)

20. Rogers L.C.G., Williams D. Diffusions, Markov Processes and Martingales, v. 2. Cambridge: Cambridge Univ. Press, 2000, 480 p.

21. Rüschendorf L., Urusov M. A. On a class of optimal stopping problems for diffusions with discontinuous coefficients. - Ann. Appl. Probab., 2008, v. 18, № 3, p 847-878.

22. Salminen P. Optimal stopping of one-dimensional diffusions. - Math. Nachr., 1985, v. 124 , p. $85-101$.

23. Ширяев A. Н. Статистический последовательный анализ: Оптимальные правила остановки. М.: Наука, 1969, 231 с.; 2-е изд., перераб. М.: Наука, 1976, 272 с. 